\title{
Texture analysis of CT imaging for assessment of esophageal squamous cancer aggressiveness
}

\author{
Song Liu ${ }^{1 *}$, Huanhuan Zheng ${ }^{1 *}$, Xia Pan ${ }^{1}$, Ling Chen ${ }^{2}$, Minke Shi ${ }^{3}$, Yue Guan ${ }^{4}$, Yun Ge ${ }^{4}, \mathrm{Jian} \mathrm{He}^{1}$, \\ Zhengyang Zhou ${ }^{1}$
}

${ }^{1}$ Department of Radiology, ${ }^{2}$ Department of Pathology, ${ }^{3}$ Department of Thoracic and Cardiovascular Surgery, Nanjing Drum Tower Hospital, The Affiliated Hospital of Nanjing University Medical School, Nanjing 210008, China; ${ }^{4}$ School of Electronic Science and Engineering, Nanjing University, Nanjing 210046, China

Contributions: (I) Conception and design: Y Ge, J He, Z Zhou; (II) Administrative support: Z Zhou; (III) Provision of study materials or patients: L Chen, M Shi, Y Guan; (IV) Collection and assembly of data: S Liu, H Zheng, X Pan; (V) Data analysis and interpretation: S Liu, H Zheng, X Pan; (VI) Manuscript writing: All authors; (VII) Final approval of manuscript: All authors.

*These authors contributed equally to this work.

Correspondence to: Yun Ge, MS, PhD. School of Electronic Science and Engineering, Nanjing University, Nanjing 210046, China. Email: geyunnju@126.com; Jian He, MD, PhD. No. 321 Zhongshan Road, Nanjing 210008, China. Email: hjxueren@126.com; Zhengyang Zhou, MD, PhD. No. 321 Zhongshan Road, Nanjing 210008, China. Email: zyzhou@nju.edu.cn.

Background: To explore the role of texture analysis of computed tomography (CT) images in preoperative assessment of esophageal squamous cell carcinoma (ESCC) aggressiveness.

Methods: Seventy-three patients with pathologically confirmed ESCC underwent unenhanced and contrast enhanced CT imaging preoperatively. Texture analysis was performed on unenhanced and contrast enhanced CT images, respectively. Six CT texture parameters were obtained. One-way analysis of variance or independent-samples t-test (normality), independent-samples Kruskal-Wallis test or Mann-Whitney U test (non-normality), binary Logistic regression analysis (multivariable), Spearman correlation test, receiver operating characteristic (ROC) curve analysis and intraclass correlation coefficient (ICC) were used for statistical analyses.

Results: Kurtosis was an independent predictor for T stages (T1-2 vs. T3-4) as well as overall stages (I-II vs. III-IV) based on unenhanced CT images, while entropy was an independent predictor for T stages (T1-2 vs. T3-4), lymph node metastasis $(\mathrm{N}-v s . \mathrm{N}+$ ) and overall stages (I/II vs. III/IV). Skew and kurtosis based on unenhanced CT images showed significant differences among $\mathrm{N}$ stages (N0, N1, N2 and N3) as well as 90th percentile based on contrast enhanced CT images. In correlation with T stage of ESCC, kurtosis and entropy significantly correlated with $\mathrm{T}$ stage both on unenhanced and contrast enhanced CT images. Reversely, entropy and 90th percentile based on contrast enhanced CT images showed significant correlations with $\mathrm{N}$ stage ( $\mathrm{r}$ : 0.526, 0.265; both $\mathrm{P}<0.05)$, as well as overall stage ( $\mathrm{r}: 0.562,0.315$; both $\mathrm{P}<0.05)$. For identifying ESCC with different T stages (T1-2 vs. T3-4), lymph node metastasis (N-vs. $\mathrm{N}+$ ) and overall stages (I/II vs. III/IV), entropy based on contrast enhanced CT images, showed good performance with area under ROC curve area under curve (AUC) of $0.637,0.815$ and 0.778 , respectively.

Conclusions: Texture analysis of CT images held great potential in differentiating different T, N and overall stages of ESCC preoperatively, while failed to assess the differentiation degrees.

Keywords: Computed tomography (CT); texture analysis; esophageal cancer; staging

Submitted Mar 01, 2017. Accepted for publication May 16, 2017.

doi: $10.21037 /$ jtd.2017.06.46

View this article at: http://dx.doi.org/10.21037/jtd.2017.06.46 


\section{Introduction}

Esophageal cancer has very high incidence and mortality rate in both more as well as less developed countries (1). The important indexes reflecting the aggressiveness of esophageal cancer include TNM stage and its differentiation degree $(2,3)$, which might be evaluated by endoscopic ultrasonography (EUS), computed tomography (CT) or endoscopic biopsy preoperatively. EUS performed well in $\mathrm{T}$ staging but poorly in $\mathrm{N}$ staging with a low sensitivity and specificity (4). In some patients with esophageal obstruction, the endoscopy even fails to go through the narrow lumen. Specimens from endoscopic biopsy are limited to achieve reliable histopathological assessment. Traditional CT could identify T4 stage disease confidently when the tumor invades into adjacent structures, but faces the challenge to differentiate early esophageal cancers due to its lower soft tissue resolution (5). CT evaluation of nodal status is mainly based on size measurement of lymph nodes, which yielded a highly discrepant sensitivity from $11 \%$ to $50 \%$ and a relatively high specificity from $83 \%$ to $95 \%$ (6-8).

Quantitative CT imaging brings new perspective into lesion's assessment (9-11). Texture analysis of CT images is one of ways, which could reflect certain components within the tumor, intratumoral heterogeneity of various tumors (12-16). For instance, Ganeshan et al. reported that CT texture analysis were associated with tumor metabolism, stage and survival of the primary esophageal lesion (16). And $\mathrm{Ha}$ et al. found that texture analysis of ${ }^{18} \mathrm{~F}-\mathrm{FDG}$ position emission tomography (PET)/CT images had significant different values between adenocarcinoma and squamous cell carcinoma for non-small cell lung carcinoma (13).

Tumor with deeper invasion and lower differentiation degree is more prone to lymph node metastasis (3). Different components within the tumor, such as hypercellular areas, necrosis, hemorrhage, and myxoid changes, can be quantified by CT texture parameters, including lower and higher percentiles (12). Furthermore, skew and kurtosis can represent the deviation from normal distribution of CT value histogram within the tumor, while entropy is able to reflect intratumoral heterogeneity quantitatively which is regarded as the degree of disorder. For instance, $\mathrm{Ng}$ et al. reported that entropy, kurtosis and skew were related to 5 -year overall survival rate in colorectal cancer patients (17). Moreover, Giganti et al. found that entropy and skew correlated with the prognosis of gastric cancer patients (18). In general, it is considered that the changes of composition, the deviation from normal distribution and the degree of disorder within tumor are closely associated with tumor aggressiveness. Thus, the above parameters have been widely investigated in the evaluation of various tumor aggressiveness $(12,17,18)$.

In recent years, CT texture analysis has also been applied in the assessment of esophageal cancer preliminary, whereas most of those studies just focused on the evaluation or prediction of treatment response in esophageal cancer (19-21). Few papers mentioned the correlation between the texture parameters based on contrast enhancement CT images and stage of esophageal cancer $(16,22)$, yet those parameters were based on PET which leads to overspending and radiation overusing. Since CT texture analysis could reflect intratumoral heterogeneity quantitatively from the perspective of non-invasive imaging, we hypothesized that with the development of esophageal cancer's aggressiveness, its biological components, microstructures as well as microenvironments would take some changes, which might be reflected by texture analysis of CT images.

Therefore, the purpose of this study was to explore the correlation between texture analysis of CT images and the aggressiveness of esophageal squamous cell carcinoma (ESCC), and to explore the role of CT texture parameters in preoperative assessment of ESCC's aggressiveness.

\section{Methods}

\section{Patients}

This retrospective study was approved by the local ethics committee and informed consent from the patients was waived. From January 2011 to January 2016, a total of 136 patients with a clinical diagnosis of ESCC were collected and reviewed. The inclusion criteria were: (I) with a diagnosis of ESCC confirmed by postoperative pathology; (II) with unenhanced and contrast enhanced CT examination preoperatively. The exclusion criteria were: (I) with any local or systematic treatment before CT examination or surgery $(27 / 63,42.9 \%)$; (II) without definite information of postoperative pathologic $\mathrm{T}$ and $\mathrm{N}$ staging (16/63, 25.4\%); (III) with a minimum diameter of tumor less than $5 \mathrm{~mm}$ insufficient to contain a region of interest (ROI) (15/63, 23.8\%); (IV) poor CT image quality for post processing due to artifacts $(5 / 63,7.9 \%)$, respectively.

The study workflow diagram with respect to patient selection is shown in Figure 1. A total of 73 patients served as our study cohort, whose clinicopathological characteristics are shown in Table 1 . 


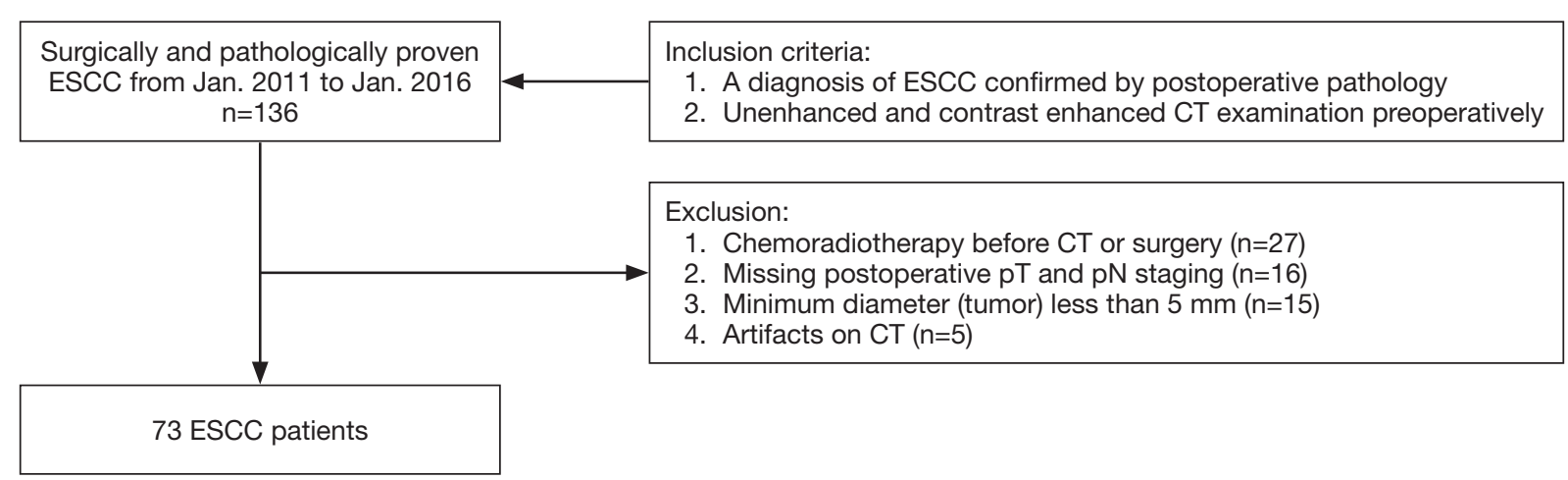

Figure 1 The study workflow diagram with respect to patient selection. ESCC, esophageal squamous cell carcinoma; pT, pathological tumor; pN, pathological node status.

Table 1 Clinicopathologic characteristics of patients with esophageal squamous carcinomas

\begin{tabular}{|c|c|c|}
\hline Characteristics & $\mathrm{n}$ & Percentage (\%) \\
\hline \multicolumn{3}{|l|}{ Gender } \\
\hline Male & 55 & 75.3 \\
\hline Female & 18 & 24.7 \\
\hline \multicolumn{3}{|c|}{ Differentiation degree } \\
\hline Poor & 10 & 13.7 \\
\hline Moderate & 45 & 61.6 \\
\hline Well & 18 & 24.7 \\
\hline \multicolumn{3}{|l|}{ T stage } \\
\hline $\mathrm{T} 1$ & 14 & 19.2 \\
\hline T2 & 23 & 31.5 \\
\hline T3 & 34 & 46.6 \\
\hline $\mathrm{T} 4$ & 2 & 2.7 \\
\hline \multicolumn{3}{|l|}{$\mathrm{N}$ stage } \\
\hline No & 37 & 50.7 \\
\hline N1 & 16 & 21.9 \\
\hline N2 & 13 & 17.8 \\
\hline N3 & 7 & 9.6 \\
\hline \multicolumn{3}{|l|}{ Overall stage } \\
\hline I & 13 & 17.8 \\
\hline II & 31 & 42.5 \\
\hline III & 28 & 38.4 \\
\hline IV & 1 & 1.4 \\
\hline
\end{tabular}

\section{CT examination}

Patients fasted for at least six hours before CT examination. The patients were trained to hold breath before CT examinations. Patients were in supine position with feet first in 16- or 64-slice multidetector CT scanners (Light Speed Pro 16, VCT, or HD Discovery 750, GE Healthcare, US). The scan ranged from the oropharynx to cardia of stomach. After unenhanced CT scan, a total of 100-120 mL iohexol (Omnipaque $350 \mathrm{mg} \mathrm{I} / \mathrm{mL}$, GE Healthcare, Shanghai, China) was administered intravenously at a flow rate of $3.0 \mathrm{~mL} / \mathrm{s}$ by using a high pressure syringe (Medrad Stellant CT Injector System; One Medrad Drive Indianola, PA, US). Contrast-enhanced CT images were obtained at one phase after 25-30 seconds following contrast agent administration. CT scan parameters were as follows: tube voltage, $120 \mathrm{kVp}$; tube current, 250-350 $\mathrm{mA}$; slice thickness, $5 \mathrm{~mm}$; slice interval, $5 \mathrm{~mm}$; field of view, 35-50 cm; matrix, $512 \times 512$; rotation time, $0.7 \mathrm{~s}$; pitch, 1.375 ; reconstruction algorithm, standard.

\section{Image interpretation and post-process}

Two radiologists with 7 - and 10-year experience in gastrointestinal imaging, who were blinded to pathologic information, performed CT image interpretation and postprocess independently. The unenhanced and contrastenhanced CT images were loaded into our in-house software (Image analyzer 1.0, China) for texture analysis. ESCC presented as thickening of esophageal wall or a mass lesion, with varying degree enhancement on contrast enhanced images. Axial unenhanced and contrast enhanced 

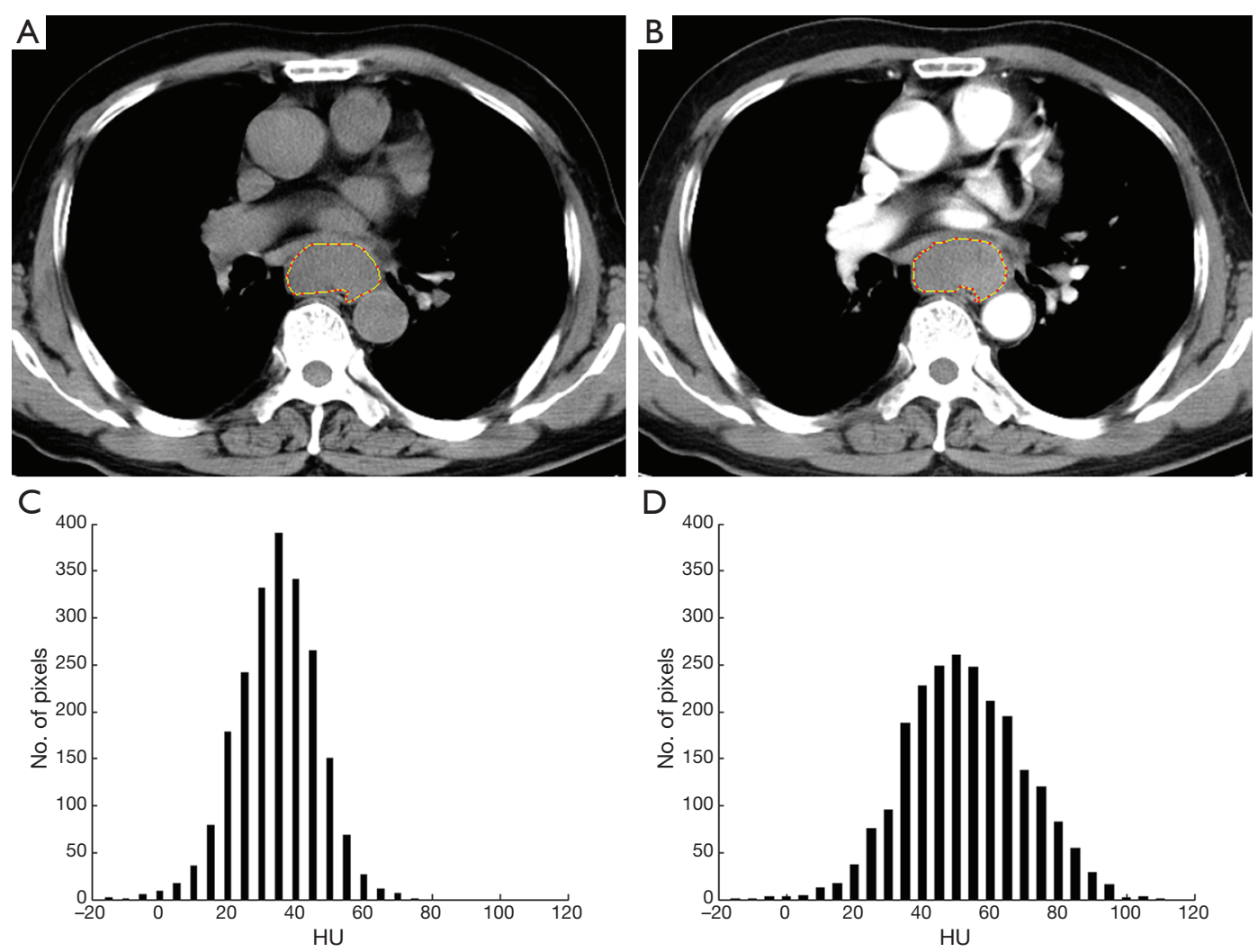

Figure 2 A 71-year-old man with pathologically diagnosed moderately differentiated squamous carcinoma at stage IIIB (T3/N2/M0). (A) Axial unenhanced CT image shows a mass in middle of the oesophagus, and the outline indicates the ROI; (B) corresponding contrast enhanced CT image shows mild enhancement of this lesion; (C) Histogram obtained from unenhanced CT image shows pixel distribution with a bin size of $5 \mathrm{HU}$; mean $=37 \mathrm{HU}, 10$ th and 90th percentile $=23$ and $50 \mathrm{HU}$, skew $=-1.57$, kurtosis $=51.14$, and entropy $=3.81$, respectively; (D) Histogram obtained from contrast enhanced CT image shows; mean $=55 \mathrm{HU}, 10$ th and 90 th percentile $=35$ and 77 HU, skew $=1.89$, kurtosis $=44.65$, and entropy $=4.17$, respectively. ROI, region of interest; HU, hounsfield units.

CT images showing the largest area of the lesion was selected. And the ROIs were manually drawn to cover the boundary of the lesion including necrosis within the tumor, carefully excluding adjacent water or air. Examples of unenhanced and contrast enhanced CT images ROIs and signal intensity histograms are shown in Figure 2. The mean area of the ROIs was $349.73 \pm 259.58$ (range, $71.51-1,376.93$ ) $\mathrm{mm}^{2}$ and $369.13 \pm 259.05$ (range, 70.34 $1,436.53) \mathrm{mm}^{2}$ in the unenhanced and contrast enhanced CT images, respectively. After drawing the ROIs, CT texture analysis was performed and six parameters based on hounsfield unit (HU) values were generated, including: (I) mean (mean HU value); (II-III) 10th and 90th percentiles (10th and 90th percentile HU value of a cumulative histogram); (IV) skew (asymmetry of the histogram distribution); (V) kurtosis (peakedness of the histogram distribution); and (VI) entropy (the irregularity of greylevel HU). Unenhanced and contrast enhanced CT images were analyzed independently. And the average value of the two radiologists was calculated for statistical analyses except interobserver agreement analysis.

\section{Pathological analysis}

Patients underwent resectable surgery by two surgeons with 18- and 10-year experience in general thoracic surgery. Histopathological analysis of the resected specimens was performed by one pathologist with 6-year experience in gastrointestinal pathology. The pathological types, differentiation degree, $\mathrm{T}$ and $\mathrm{N}$ stage of the specimens were reviewed and recorded according to the American Joint Committee on Cancer (7th edition). 


\section{Statistical analyses}

Shapiro-Wilk tests were used to check normality assumption for all parameters. The results showed in Tables S1-S5. Firstly, Independent samples $t$-test (normality) or MannWhitney U test (non-normality) was used to compare texture parameters between different $\mathrm{T}$ stages (T1-2 vs. T3-4), lymph node metastasis $(\mathrm{N}-v s . \mathrm{N}+$ ) and overall stages (I/II vs. III/IV). Secondly, the texture parameters which were significant in Independent samples $t$-test (normality) or Mann-Whitney U test (non-normality) were tested by binary Logistic regression analysis (multivariable) to identify the above pathological characteristics. Then, diagnostic performance of CT texture parameters which were significant in logistic regression analysis in differentiating the above pathological characteristics with receiver operating characteristic (ROC) curve analysis. One-way analysis of variance (normality) or independent-samples Kruskal-Wallis test (non-normality) was used to detect the difference of texture parameters among different $\mathrm{N}(\mathrm{N} 0, \mathrm{~N} 1, \mathrm{~N} 2$ and N3) and differentiation degrees (poor, moderate and well). Correlations between CT texture parameters and T, N, and overall stages as well as differentiation degrees were analyzed with Spearman correlation test ( $\mathrm{r}, 0.000-0.300$, poor; 0.301-0.500, moderate; 0.501-0.800, good; 0.801-1.000, excellent). Interobserver agreement in measurement of CT texture parameters was evaluated by calculating intraclass correlation coefficient (ICC) $(0.000-0.200$, poor; 0.201-0.400, fair; 0.401-0.600, moderate; 0.601-0.800, good; 0.801-1.000, excellent). Statistical analyses were performed with SPSS (version 22.0 for Microsoft Windows x64, SPSS, Chicago, US). A two-tailed P value less than 0.05 was considered statistically significant.

\section{Results}

\section{Differences of CT texture parameters among different pathologic characteristics in ESCCs}

According to independent samples $t$-test (normality) or Mann-Whitney $U$ test (non-normality), multiple texture parameters have significant differences between different T stages (T1/2 vs. T3/4) and overall stages (I/II vs. III/IV) based on unenhanced and contrast enhanced CT images (both $\mathrm{P}<0.05)$ (Table S6). And binary logistic regression analysis (multivariable) showed that entropy based on contrast enhanced CT images was independent predictor for $\mathrm{T}$ stage ( $\mathrm{T} 1-2$ vs. $\mathrm{T} 3-4 ; \mathrm{P}=0.030)$, lymph node metastasis $(\mathrm{N}-v s . \mathrm{N}+; \mathrm{P}=0.001)$ and overall stage
(I-II vs. III-IV; $\mathrm{P}=0.001)$. Entropy and kurtosis based on unenhanced CT images were independent predictors for T stage (T1-2 vs. T3-4; $\mathrm{P}=0.035,0.026$, respectively). And kurtosis based on unenhanced CT images was independent predictor for overall stage (I-II vs. III-IV; $\mathrm{P}=0.020$ ) (Table S7). There were significant differences of skew and kurtosis based on unenhanced CT images among ESCCs at different $\mathrm{N}$ stages using one-way analysis of variance (normality) or independent-samples Kruskal-Wallis test (non-normality), as well as 90th percentile and entropy based on contrast enhanced CT images $(\mathrm{P}<0.05)$. There were no significant differences of all parameters at different differentiation degrees of ESCCs (all $\mathrm{P}>0.05$ ) (Table S8).

\section{Correlations between CT texture parameters and tumor stages as well as differentiation degrees}

Based on unenhanced CT texture analysis, kurtosis and entropy correlated with T stages of ESCC significantly (r: 0.418, 0.283; both $\mathrm{P}<0.05$ ). Mean, 10th-90th percentile, skew and kurtosis, correlated with both $\mathrm{N}$ and overall stages significantly (r: -0.261 to $0.309,-0.273$ to 0.530 ; all $\mathrm{P}<0.05$ ) (Table 2).

Based on contrast enhanced CT texture analysis, kurtosis and entropy significantly correlated with $\mathrm{T}$ stage (r: 0.277 , $0.267 ; \mathrm{P}=0.018,0.022$, respectively). The 90 th percentile and entropy correlated with both $\mathrm{N}$ and overall stages of ESCC significantly (r: $0.265,0.526 ; \mathrm{r}: 0.315,0.562$, respectively; both $\mathrm{P}<0.05$ ) (Table 2).

No parameters showed significant correlation with differentiation degrees in ESCC both on unenhanced and contrast enhanced CT $(\mathrm{P}>0.05)$ (Table 2).

\section{Diagnostic performance for differentiating pathological characteristics}

For identifying ESCC with different T stages (T1-2 vs. T3-4), lymph node metastasis $(\mathrm{N}-v s . \mathrm{N}+$ ) and overall stages (I-II $v s$. III-IV), the results of ROC curve analysis were shown in Figure 3.

Based on unenhanced CT images, kurtosis and entropy could differentiate different $\mathrm{T}$ stages (T1-2 vs. T3-4), with an area under curve (AUC) of 0.652 and 0.653 , respectively. Kurtosis identified different overall stages (I-II vs. III-IV) with an AUC of 0.744.

Based on contrast enhanced CT images, entropy differentiated different $\mathrm{T}$ stages (T1-2 vs. T3-4), lymph node metastasis $(\mathrm{N}-v s . \mathrm{N}+$ ) and overall stages (I/II vs. III/IV) 
Table 2 Correlations between unenhanced CT texture parameters and tumor stage of esophageal squamous carcinoma

\begin{tabular}{|c|c|c|c|c|c|c|c|c|}
\hline Parameters & \multicolumn{2}{|c|}{ T stage } & \multicolumn{2}{|c|}{$\mathrm{N}$ stage } & \multicolumn{2}{|c|}{ Overall stage } & \multicolumn{2}{|c|}{ Differentiation degree } \\
\hline \multicolumn{9}{|l|}{ Unenhanced CT } \\
\hline Mean & -0.099 & 0.406 & -0.309 & $0.008^{*}$ & -0.306 & $0.008^{\star}$ & 0.073 & 0.538 \\
\hline 10th percentile & -0.211 & 0.331 & -0.287 & $0.014^{*}$ & -0.273 & $0.019^{*}$ & 0.090 & 0.447 \\
\hline Skew & -0.164 & 0.165 & -0.261 & $0.026^{*}$ & -0.401 & $<0.001^{*}$ & -0.071 & 0.552 \\
\hline Kurtosis & 0.418 & $<0.001^{*}$ & 0.309 & $0.008^{*}$ & 0.530 & $<0.001^{*}$ & 0.090 & 0.450 \\
\hline Entropy & 0.283 & $0.015^{\star}$ & 0.120 & 0.312 & 0.169 & 0.154 & -0.019 & 0.876 \\
\hline \multicolumn{9}{|l|}{ Enhanced CT } \\
\hline 90th percentile & 0.073 & 0.538 & 0.265 & $0.024^{*}$ & 0.315 & $0.007^{\star}$ & -0.042 & 0.724 \\
\hline Skew & 0.018 & 0.888 & -0.149 & 0.209 & 0.027 & 0.818 & -0.124 & 0.298 \\
\hline Kurtosis & 0.277 & $0.018^{\star}$ & 0.073 & 0.539 & 0.192 & 0.104 & 0.072 & 0.545 \\
\hline Entropy & 0.267 & $0.022^{*}$ & 0.526 & $<0.001^{*}$ & 0.562 & $<0.001^{\star}$ & -0.034 & 0.773 \\
\hline
\end{tabular}

*, $\mathrm{P}<0.05$ with Spearman correlation test. CT, computed tomography.

with an AUC of $0.637,0.815$ and 0.778 , respectively.

Mean and 10th percentiles based on unenhanced CT images, and entropy based on contrast enhanced CT images showed excellent interobserver agreement with ICCs over 0.800 (Table S9).

\section{Discussion}

We confirmed the correlations between CT texture parameters and T, N and overall stage of ESCC in this study.

We found that kurtosis and entropy based on unenhanced CT images differed significantly among ESCCs at different T stages (T1/2 vs. T3/4) as well as entropy based contrast enhanced CT images. Moreover, kurtosis and entropy significantly correlated with $\mathrm{T}$ stage both on unenhanced and contrast enhanced CT images. Kurtosis reflected the peakedness degree of the pixel density histogram and entropy represented the degree of tumor chaos and complexity. With progression of $\mathrm{T}$ stage, the $\mathrm{CT}$ values within the tumor became more heterogeneous, the distribution of the pixel density form deviated from the normal distribution, so the value of kurtosis and entropy became larger. In our study, some parameters (mean, 10th, 90th percentile and skew) were unreliable for $\mathrm{T}$ stage of ESCC both in unenhanced and contrast enhanced CT.

Our study showed that entropy differed significantly among ESCCs at different $\mathrm{N}$ stages, based on contrast enhanced CT image $(\mathrm{P}<0.001)$, while there were no significant differences on unenhanced $\mathrm{CT}$ image $(\mathrm{P}>0.05)$. In correlation with $\mathrm{N}$ stage of ESCC, entropy based on contrast enhanced CT images $(r: 0.526 ; \mathrm{P}<0.001)$ was also superior to unenhanced CT images $(\mathrm{P}>0.05)$. Entropyreflected the heterogeneity of the pixel density in CT images. In contrast enhanced CT images, entropy represented the heterogeneity of the tumor's blood supply. We found that as the heterogeneity of blood supply with the tumor increased, its potential of lymph node metastasis also increased. There was a report that metabolic parameters of non-small cell lung carcinoma correlated with entropy assessed by texture analysis of FDG-PET images (23). The correlation between some parameters (mean, 10th, 90th percentile, skew and kurtosis) based on the unenhanced CT images and N stage of ESCC was weak, as well as 90th percentile based on the contrast enhanced CT images in our study.

It was interesting to found that most texture parameters 

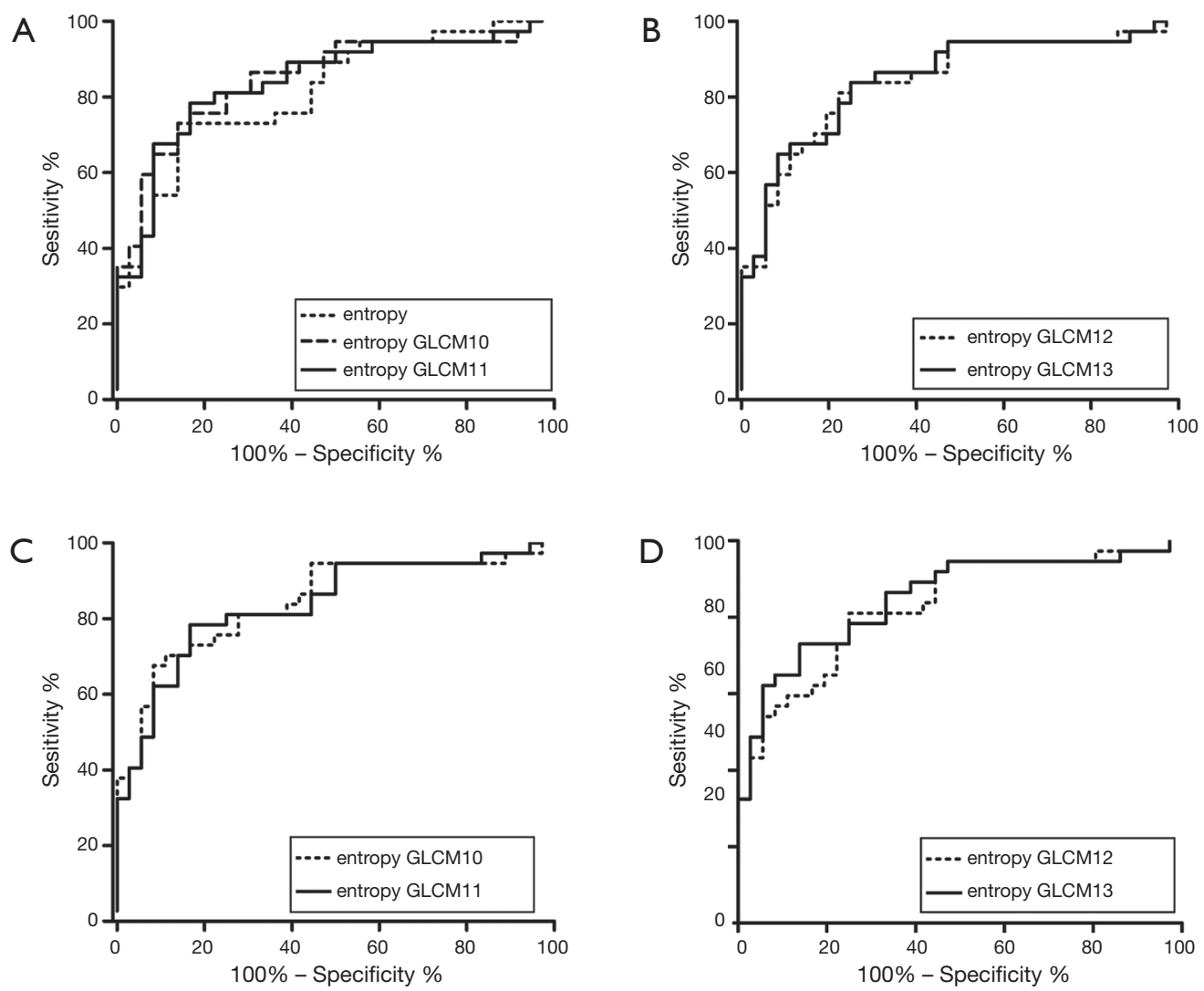

Figure 3 ROC curves of texture parameters derived from CT images in differentiating different pathological characteristics of ESCCs. (A) ROC curves of kurtosis and entropy based on unenhanced CT images in differentiating different T stages (T1-2 vs. T3-4), with an AUC of 0.652 and 0.653 , respectively; (B) ROC curve of entropy based on contrast enhanced CT images differentiating different $\mathrm{T}$ stages (T1-2 vs. T3-4) with an AUC of 0.637; (C) ROC curve of entropy based on contrast enhanced CT images in differentiating lymph node metastasis $(\mathrm{N}-v s . \mathrm{N}+)$ with an AUC of 0.815; (D) ROC curves of kurtosis based on unenhanced CT images and entropy based on contrast enhanced CT images in differentiating overall stages (I/II vs. III/IV) with an AUC of 0.744 and 0.778 , respectively. ROC, receiver operating characteristic; ESCC, esophageal squamous cell carcinoma; AUC, area under curve.

which correlated with $\mathrm{N}$ stage were also correlated with overall stage. Nevertheless, their correlation with overall stage was weaker than $\mathrm{N}$ stage but stronger than $\mathrm{T}$ stage, which might be due to a higher weight of $\mathrm{N}$ stage than $\mathrm{T}$ stage in overall stage. In our study, energy based on contrast enhanced CT images significantly correlated with overall stage of ESCC, which was in accordance with the results of some previous studies. For example, Dong et al. reported that entropy and energy based on PET images correlated significantly with ESCC stage according to AJCC 7th (22). Ganeshan et al. also found that entropy showed significant differences between patients with esophageal cancers at stages I-II and III-IV (16).

However, we failed to detect any significant differences of CT texture parameters among ESCCs with different differentiation degrees. Dong et al. also reported that no significant correlation was found between texture parameter and histological grade (22). Hence, the role of texture analysis on CT or PET images in evaluating differentiation degree of ESCC remains undetermined.

Entropy based on contrast enhanced CT images performed better than unenhanced CT images in identifying ESCCs with lymph node metastasis and overall stages (I-II vs. III-IV), especially for lymph node metastasis (AUC $=0.815$ ). Many studies have highlighted the value of entropy in differentiating positive-human papillomavirus form negative-human papillomavirus primary oropharyngeal squamous cell (24), identifying colorectal cancer patients with metastatic liver disease (25), and predicting lung cancer recurrence after stereotactic ablative radiotherapy (26). 
Most parameters showed good or excellent interobserver agreement, with ICCs ranging from 0.699 to 0.921 , indicating their stability. While a few parameters showed poor or fair interobserver agreement with ICCs ranging from 0.336 to 0.509 . For example, skew showed the worst interobserver agreement (ICC $=0.336$ ) which was not suitable for evaluating CT texture images of ESCC.

Our study had some limitations. Firstly, patients of ESCC at stage M1 were not included due to lack of postoperative pathologic information, which might cause some selection bias to our study. Secondly, the ROIs were manually drawn on CT without reference to pathologic specimens, and only one slice showing the largest area of the lesion was selected rather than whole lesion analysis. Thirdly, CT texture analysis was only performed on primary tumors rather than lymph nodes, which might compromise the prediction efficiency. Finally, CT images were obtained from two CT scanners which might affect the texture analysis, while a previous study showed the effect of variations in acquisition parameters of CT may be limited when performing texture analyses (27). All the above issues required further investigation.

In conclusion, our study demonstrated that texture analysis on CT images could help to evaluate preoperative staging of ESCC, especially kurtosis and entropy based on unenhanced images for $\mathrm{T}$ staging and entropy based on contrast enhanced images for $\mathrm{N}$ and overall staging. And CT texture parameters proved useful in assessing ESCC's aggressiveness, which might facilitate personalized treatment and improve the prognosis of patients with ESCC.

\section{Acknowledgements}

None.

\section{Footnote}

Conflicts of Interest: The authors have no conflicts of interest to declare.

Ethical Statement: This retrospective study was approved by the local ethics committee (No. 2016-183-1) and informed consent from the patients was waived.

\section{References}

1. Gupta B, Kumar N. Worldwide incidence, mortality and time trends for cancer of the oesophagus. Eur J Cancer Prev 2017;26:107-18.

2. Huang Y, Guo W, Shi S, et al. Evaluation of the 7(th) edition of the UICC-AJCC tumor, node, metastasis classification for esophageal cancer in a Chinese cohort. J Thorac Dis 2016;8:1672-80.

3. Ma H, Li Y, Ding Z, et al. The clinical significance of subcarinal lymph node dissection in the radical resection of oesophageal cancer. Interact Cardiovasc Thorac Surg 2013;16:839-43.

4. Sun F, Chen T, Han J, et al. Staging accuracy of endoscopic ultrasound for esophageal cancer after neoadjuvant chemotherapy: a meta-analysis and systematic review. Dis Esophagus 2015;28:757-71.

5. Griffin Y. Esophageal Cancer: Role of Imaging in Primary Staging and Response Assessment Post Neoadjuvant Therapy. Semin Ultrasound CT MR 2016;37:339-51.

6. Yoon YC, Lee KS, Shim YM, et al. Metastasis to regional lymph nodes in patients with esophageal squamous cell carcinoma: CT versus FDG PET for presurgical detection prospective study. Radiology 2003;227:764-70.

7. van Vliet EP, Heijenbrokkal MH, Hunink MG, et al. Staging investigations for oesophageal cancer: a metaanalysis. Br J Cancer 2008;98:547.

8. Lee G, Kim SJ, Jeong YJ, et al. Clinical implication of PET/MR imaging in preoperative esophageal cancer staging: comparison with PET/CT, endoscopic ultrasonography, and CT. J Nucl Med 2014;55:1242-7.

9. Epstein ML, Obara PR, Chen Y, et al. Quantitative radiology: automated measurement of polyp volume in computed tomography colonography using Hessian matrix-based shape extraction and volume growing. Quant Imaging Med Surg 2015;5:673-84.

10. Cavalcanti PG, Shirani S, Scharcanski J, et al. Lung nodule segmentation in chest computed tomography using a novel background estimation method. Quant Imaging Med Surg 2016;6:16-24.

11. Grenier PA, Fetita CI, Brillet PY. Quantitative computed tomography imaging of airway remodeling in severe asthma. Quant Imaging Med Surg 2016;6:76-83.

12. Reiner CS, Gordic S, Puippe G, et al. Histogram Analysis of CT Perfusion of Hepatocellular Carcinoma for Predicting Response to Transarterial Radioembolization: Value of Tumor Heterogeneity Assessment. Cardiovasc Intervent Radiol 2016;39:400-8.

13. Ha S, Choi H, Cheon GJ, et al. Autoclustering of Nonsmall Cell Lung Carcinoma Subtypes on (18)F-FDG PET Using Texture Analysis: A Preliminary Result. Nucl Med 
Mol Imaging 2014;48:278-86.

14. Hodgdon T, McInnes MD, Schieda N, et al. Can Quantitative CT Texture Analysis be Used to Differentiate Fat-poor Renal Angiomyolipoma from Renal Cell Carcinoma on Unenhanced CT Images? Radiology 2015;276:787-96.

15. Dennie C, Thornhill R, Sethi-Virmani V, et al. Role of quantitative computed tomography texture analysis in the differentiation of primary lung cancer and granulomatous nodules. Quant Imaging Med Surg 2016;6:6-15.

16. Ganeshan B, Skogen K, Pressney I, et al. Tumour heterogeneity in oesophageal cancer assessed by CT texture analysis: preliminary evidence of an association with tumour metabolism, stage, and survival. Clin Radiol 2012;67:157-64.

17. Ng F, Ganeshan B, Kozarski R, et al. Assessment of primary colorectal cancer heterogeneity by using wholetumor texture analysis: contrast-enhanced CT texture as a biomarker of 5-year survival. Radiology 2013;266:177-84.

18. Giganti F, Antunes S, Salerno A, et al. Gastric cancer: texture analysis from multidetector computed tomography as a potential preoperative prognostic biomarker. Eur Radiol 2017;27:1831-9.

19. Yip SS, Coroller TP, Sanford NN, et al. Use of registration-based contour propagation in texture analysis for esophageal cancer pathologic response prediction. Phys Med Biol 2016;61:906-22.

20. Yip SS, Coroller TP, Sanford NN, et al. Relationship between the Temporal Changes in Positron-EmissionTomography-Imaging-Based Textural Features and Pathologic Response and Survival in Esophageal Cancer Patients. Front Oncol 2016;6:72.

Cite this article as: Liu S, Zheng H, Pan X, Chen L, Shi M, Guan Y, Ge Y, He J, Zhou Z. Texture analysis of CT imaging for assessment of esophageal squamous cancer aggressiveness. J Thorac Dis 2017;9(11):4724-4732. doi: 10.21037/ jtd.2017.06.46
21. Yip C, Davnall F, Kozarski R, et al. Assessment of changes in tumor heterogeneity following neoadjuvant chemotherapy in primary esophageal cancer. Dis Esophagus 2015;28:172-9.

22. Dong X, Xing L, Wu P, et al. Three-dimensional positron emission tomography image texture analysis of esophageal squamous cell carcinoma: relationship between tumor $18 \mathrm{~F}$-fluorodeoxyglucose uptake heterogeneity, maximum standardized uptake value, and tumor stage. Nucl Med Commun 2013;34:40-6.

23. van Gomez Lopez O, Garcia Vicente AM, Honguero Martinez AF, et al. Heterogeneity in $\left[{ }^{18} \mathrm{~F}\right]$ fluorodeoxyglucose positron emission tomography/ computed tomography of non-small cell lung carcinoma and its relationship to metabolic parameters and pathologic staging. Mol Imaging 2014;13.

24. Buch K, Fujita A, Li B, et al. Using Texture Analysis to Determine Human Papillomavirus Status of Oropharyngeal Squamous Cell Carcinomas on CT. AJNR Am J Neuroradiol 2015;36:1343-8.

25. Rao SX, Lambregts DM, Schnerr RS, et al. Whole-liver CT texture analysis in colorectal cancer: Does the presence of liver metastases affect the texture of the remaining liver? United European Gastroenterol J 2014;2:530-8.

26. Mattonen SA, Palma DA, Haasbeek CJ, et al. Early prediction of tumor recurrence based on CT texture changes after stereotactic ablative radiotherapy (SABR) for lung cancer. Med Phys 2014;41:033502.

27. Miles KA, Ganeshan B, Griffiths MR, et al. Colorectal cancer: texture analysis of portal phase hepatic CT images as a potential marker of survival. Radiology 2009;250:444-52. 


\section{Supplementary}

Table S1 The P values of CT texture parameters among esophageal squamous carcinomas at different T stages for Shapiro-Wilk tests of normality assumption

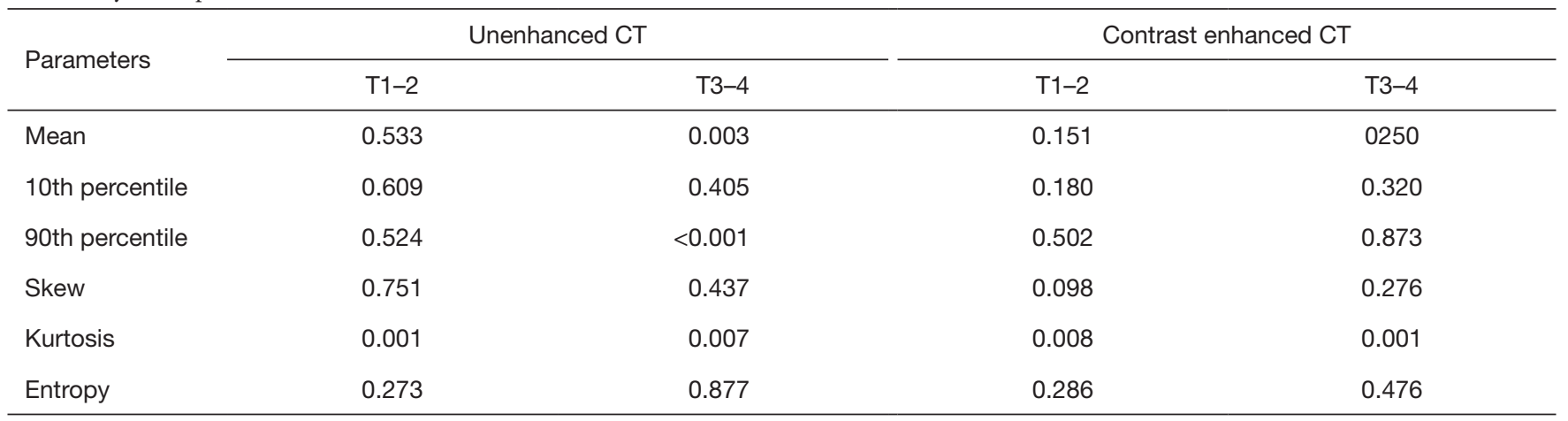

The parameters verify normality assumption if the $\mathrm{P}$ value is larger than 0.05 . T1-2, T1 and T2 stages were categorized as $\mathrm{T} 1-2$ group;

T3-4, T3 and T4 stages were categorized as T3-4 group. CT, computed tomography.

Table S2 P values of CT texture parameters among esophageal squamous carcinomas with lymph node metastasis for Shapiro-Wilk tests of normality assumption

\begin{tabular}{lcccc}
\hline \multirow{2}{*}{ Parameters } & \multicolumn{2}{c}{ Unenhanced CT } & \multicolumn{2}{c}{ Contrast enhanced CT } \\
\cline { 2 - 4 } \cline { 3 - 5 } Mean & $\mathrm{N}-$ & 0.036 & $\mathrm{~N}-$ & 0.590 \\
10th percentile & 0.351 & 0.388 & 0.226 & 0.343 \\
90th percentile & 0.294 & $<0.001$ & 0.472 & 0.896 \\
Skew & 0.528 & 0.415 & 0.027 & 0.950 \\
Kurtosis & 0.649 & 0.003 & 0.001 & $<0.001$ \\
Entropy & $<0.001$ & 0.538 & 0.272 & 0.085 \\
\hline
\end{tabular}

The parameters verify normality assumption if the $\mathrm{P}$ value is larger than $0.05 . \mathrm{N}-$, without lymph node metastasis; $\mathrm{N}+$, with lymph node metastasis; CT, computed tomography.

Table S3 P values of CT texture parameters among esophageal squamous carcinomas at different overall stages for Shapiro-Wilk tests of normality assumption

\begin{tabular}{lllll}
\hline \multirow{2}{*}{ Parameters } & \multicolumn{3}{c}{ Unenhanced CT } & \multicolumn{2}{c}{ Contrast enhanced CT } \\
\cline { 2 - 5 } \cline { 3 - 5 } Mean & I-II & III-IV & I-II & 0.575 \\
10th percentile & 0.535 & 0.138 & 0.478 & 0.805 \\
90th percentile & 0.643 & 0.561 & 0.657 & 0.914 \\
Skew & 0.489 & 0.003 & 0.001 & $<0.001$ \\
Kurtosis & 0.926 & 0.323 & 0.001 & $<0.001$ \\
Entropy & 0.001 & 0.001 & 0.305 & 0.195 \\
\hline
\end{tabular}

The parameters verify normality assumption if the P value is larger than 0.05. I-II: I and II stages were categorized as I-II group; III-IV: III and IV stages were categorized as III-IV group; CT, computed tomography. 
Table S4 P values of CT texture parameters among esophageal squamous carcinomas at different differentiation degrees for Shapiro-Wilk tests of normality assumption

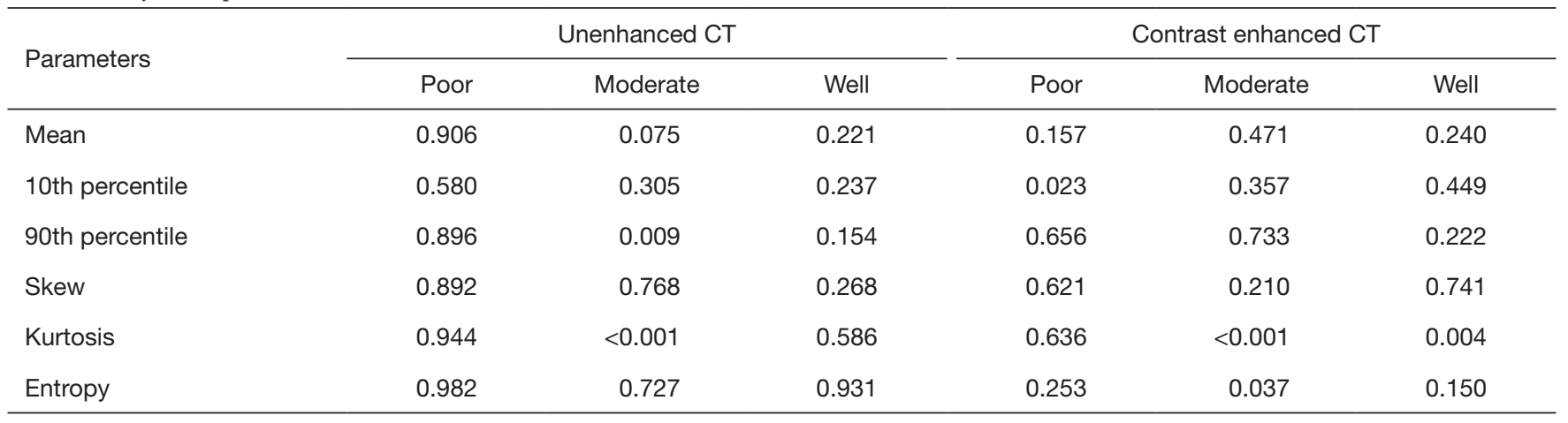

The parameters verify normality assumption if the $P$ value is larger than 0.05 . CT, computed tomography.

Table S5 P values of CT texture parameters among esophageal squamous carcinomas at different N stages for Shapiro-Wilk tests of normality assumption

\begin{tabular}{|c|c|c|c|c|c|c|c|c|}
\hline Parameters & \multicolumn{4}{|c|}{ Unenhanced CT } & \multicolumn{4}{|c|}{ Contrast enhanced CT } \\
\hline Mean & 0.351 & 0.924 & 0.016 & 0.365 & 0.590 & 0.959 & 0.981 & 0.337 \\
\hline 10th percentile & 0.294 & 0.734 & 0.019 & 0.351 & 0.226 & 0.535 & 0.862 & 0.411 \\
\hline 90th percentile & 0.528 & 0.855 & 0.002 & 0.176 & 0.472 & 0.245 & 0.686 & 0.290 \\
\hline Kurtosis & $<0.001$ & 0.136 & 0.050 & 0.490 & 0.001 & 0.001 & 0.065 & 0.034 \\
\hline Entropy & 0.178 & 0.854 & 0.800 & 0.072 & 0.272 & 0.159 & 0.964 & 0.407 \\
\hline
\end{tabular}

The parameters verify normality assumption if the $P$ value is larger than 0.05 . CT, computed tomography.

Table S6 CT texture parameters for variance comparison in different T, lymph node metastasis and overall stages of esophageal squamous carcinomas

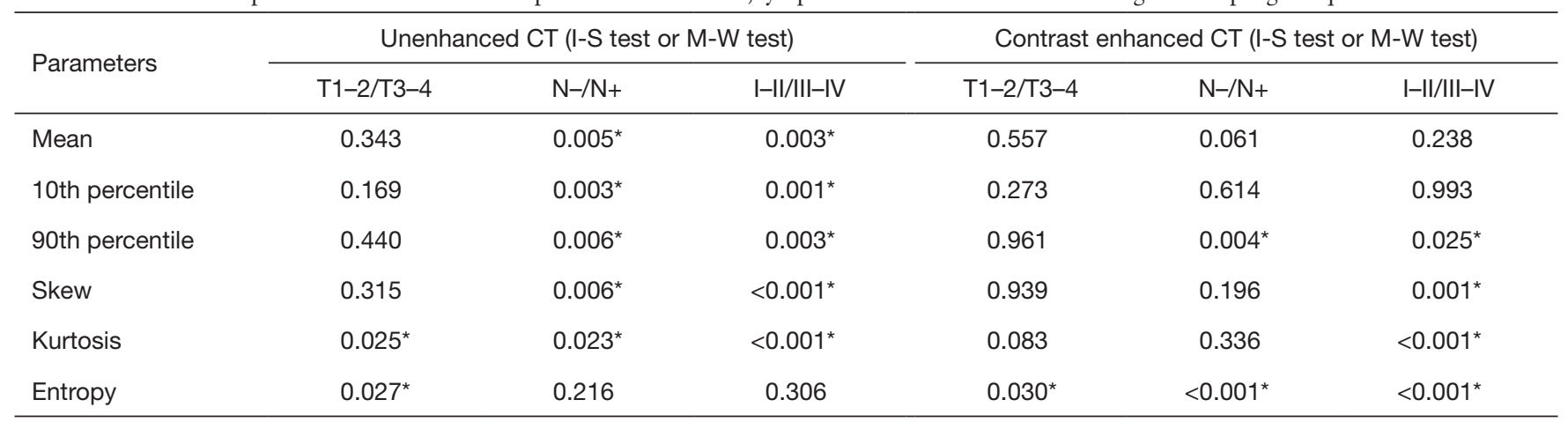

I-S test, independent samples t-test; $\mathrm{M}-\mathrm{W}$ test, Mann-Whitney $\mathrm{U}$ test. *, $\mathrm{P}<0.05 . \mathrm{T} 1-2, \mathrm{~T} 1$ and T2 stages were categorized as T1-2 group; $\mathrm{N}-/ \mathrm{N}+$ : $\mathrm{N}-$, without lymph node metastasis and $\mathrm{N}+$, with lymph node metastasis; T3-4, T3 and T4 stages were categorized as T3-4 group; $\mathrm{CT}$, computed tomography. 
Table S7 P values of Binary Logistic regression analysis for CT texture parameters which were significant in Independent samples t-test or Mann-Whitney U test

\begin{tabular}{|c|c|c|c|c|c|c|}
\hline Parameters & \multicolumn{3}{|c|}{ Unenhanced CT } & \multicolumn{3}{|c|}{ Contrast enhanced CT } \\
\hline Kurtosis & 0.035 & - & 0.020 & - & - & - \\
\hline Entropy & 0.026 & - & - & 0.030 & 0.001 & 0.001 \\
\hline
\end{tabular}

$\mathrm{T} 1-2, \mathrm{~T} 1$ and $\mathrm{T} 2$ stages were categorized as $\mathrm{T} 1-2$ group; $\mathrm{N}-/ \mathrm{N}+, \mathrm{N}-$ without lymph node metastasis and $\mathrm{N}+$ with lymph node metastasis;

T3-4, T3 and T4 stages were categorized as T3-4 group. CT, computed tomography.

Table S8 CT texture parameters for variance comparison in different N stages and differentiation degrees of esophageal squamous carcinomas

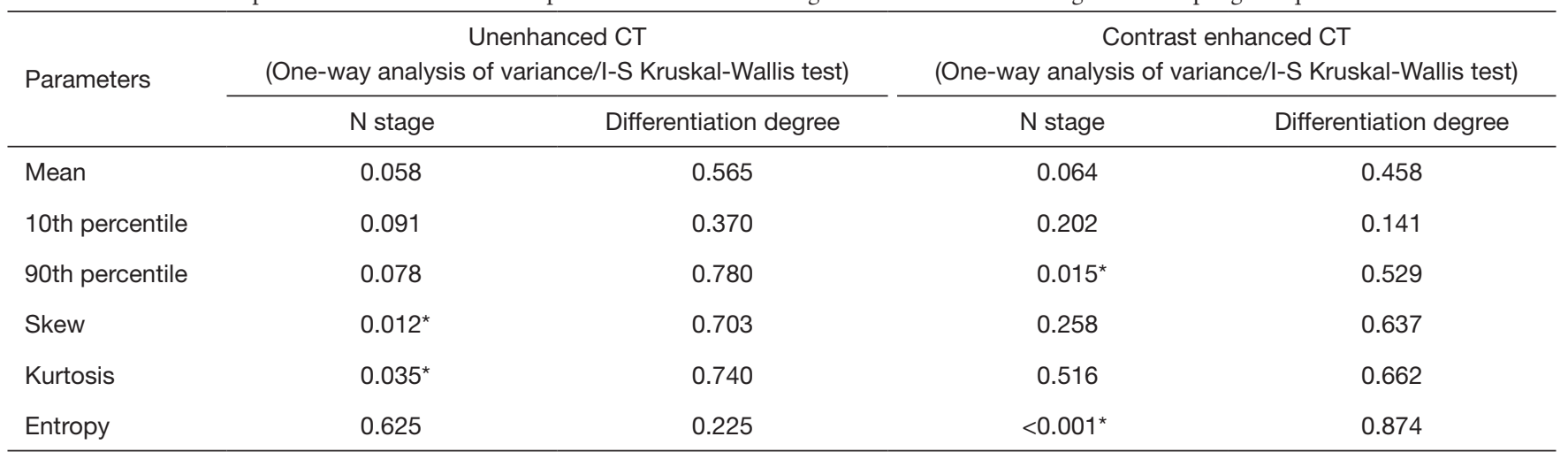

One-way analysis of variance (normality); I-S Kruskal-Wallis test, independent-samples Kruskal-Wallis test (non-normality); ${ }^{*}, \mathrm{P}<0.05$. They were used to detect the difference of texture parameters among different N stages (N0, N1, N2 and N3) and differentiation degrees (poor, moderate and well).

Table S9 Interobserver agreement of texture parameters of esophageal squamous carcinomas based on unenhanced and contrast enhanced CT images

\begin{tabular}{|c|c|c|c|c|}
\hline Parameters & \multicolumn{2}{|c|}{ Unenhanced CT } & \multicolumn{2}{|c|}{ Contrast enhanced CT } \\
\hline Mean & $0.876^{*}$ & $0.766-0.934$ & 0.726 & $0.483-0.855$ \\
\hline 10th percentile & $0.921^{*}$ & $0.851-0.958$ & 0.763 & $0.553-0.875$ \\
\hline 90th percentile & 0.772 & $0.569-0.880$ & 0.743 & $0.514-0.864$ \\
\hline Kurtosis & 0.509 & $0.072-0.740$ & 0.708 & $0.448-0.846$ \\
\hline Entropy & 0.699 & $0.431-0.841$ & $0.882^{*}$ & $0.776-0.937$ \\
\hline
\end{tabular}

${ }^{*}$, ICC $>0.800$, indicating an excellent interobserver agreement. ICC, intraclass correlation coefficient. 